\title{
Analysis of Long Noncoding RNA and mRNA Expression Profiles in IL-9-Activated Astrocytes and EAE Mice
}

\author{
Xiaomei Liu, ${ }^{a, b}$ Qing Zhang ${ }^{c}$ Weixiao Wang ${ }^{b}$ Dongjiao Zuo ${ }^{b}$ Jing Wang ${ }^{b}$ \\ Feng Zhou ${ }^{\mathrm{b}}$ Liping Niu ${ }^{\mathrm{b}}$ Xiangyang Li $^{\mathrm{b}}$ Suping Qin ${ }^{\mathrm{b}}$ Yanbo Kou ${ }^{\mathrm{b}}$ \\ Fanyun Kong ${ }^{b}$ Wei Pan ${ }^{b}$ Yugang Wang ${ }^{b}$ Dianshuai Gao ${ }^{d}$ Hong Sun ${ }^{e}$ \\ Jessica M. Meves ${ }^{f}$ Kuiyang Zheng ${ }^{b}$ Renxian Tang ${ }^{b}$ \\ ajiangsu Key Laboratory of New Drug Research and Clinical Pharmacy, Xuzhou Medical University, \\ Xuzhou, Jiangsu, bJiangsu Key Laboratory of Immunity and Metabolism, Department of Pathogen \\ Biology and Immunology and Laboratory of Infection and Immunity, Xuzhou Medical University, \\ Xuzhou, Jiangsu, 'Clinical Laboratory of Xuzhou Tumor Hospital, Xuzhou, Jiangsu, 'Department of \\ Neurobiology and Anatomy, Xuzhou Medical University, Xuzhou, Jiangsu, ${ }^{e}$ Department of Physiology, \\ Xuzhou Medical University, Xuzhou, Jiangsu, China; 'Department of Neurosciences, University of \\ California San Diego, School of Medicine, La Jolla, CA, USA
}

\section{Key Words}

Multiple sclerosis • Experimental autoimmune encephalomyelitis $\bullet$ LncRNAs • Astrocytes $\bullet$ IL-9

\begin{abstract}
Background/Aims: Multiple sclerosis (MS) is an autoimmune disease in the central nervous system associated with demyelination and axonal injury. Astrocyte activation is involved in the pathogenesis of MS and experimental autoimmune encephalomyelitis (EAE), an animal model of MS. This study was designed to find potential IncRNAs in EAE mice and activated astrocytes. Methods: we performed microarray analysis of IncRNAs from the brain tissues of EAE mice and primary mouse astrocytes treated with IL-9(50 ng/ml). 12 IncRNAs were validated through real-time PCR. Gene ontology and KEGG pathway analysis were applied to explore the potential functions of IncRNAs. Results: Differentially expressed 3300 IncRNAs and 3250 mRNAs were in the brain tissues of EAE mice, and 3748 IncRNAs and 3332 mRNAs were in activated astrocytes. Notably, there were 2 co-up-regulated IncRNAs and 3 co-downregulated IncRNAs both in the brain tissues of EAE mice and in activated astrocytes, including Gm14005, Gm12478, mouselincRNA1117, AK080435, and mouselincRNA0681, which regulate the ER calcium flux kinetics, zinc finger protein and cell apoptosis. Similarly, there were 7 mRNAs co-up-regulated and 2 mRNAs co-down-regulated both in vivo and in vitro. Gene ontology and KEGG pathway analysis showed that the biological functions of differentially expressed mRNAs were associated with metabolism, development and inflammation. The results of realtime PCR validation were consistent with the data from the microarrays. Conclusions: Our X. Liu and Q. Zhang contributed equally tot this work.

Kuiyang Zheng

and Renxian Tang


data uncovered the expression profiles of IncRNAs and mRNAs in vivo and in vitro, which may help delineate the mechanisms of astrocyte activation during MS/EAE process.

(C) 2018 The Author(s)

Published by S. Karger AG, Basel

\section{Introduction}

Multiple sclerosis (MS) is a devastating disease of the central nervous system (CNS), and is characterized by activated autoreactive $\mathrm{CD} 4^{+} \mathrm{T}$ cells that infiltrate into the CNS and mediate inflammation, demyelination, progressive axonal degradation and reactive astrogliosis $[1,2]$. MS and its animal model, experimental autoimmune encephalomyelitis (EAE), had initially been thought to be Th1-mediated diseases. Recent studies provide strong evidence that Th17 and Th9 cells also play important roles in the pathogenesis of MS/EAE [3]. IL-9, a signature cytokine produced by Th9 and Th17 cells, is able to amplify Th17 development via a positive feedback loop [3]. Furthermore, IL-9 promotes Th17 cell migration into the CNS through the activation of astrocytes, and blocking IL-9 with neutralizing antibodies ameliorates the EAE $[4,5]$.

Astrocytes, the major glial cell type within the CNS, regulate neuronal function and participate in the formation of the blood-brain barrier (BBB) [6]. Furthermore, astrocytes have the capacity to interact with the peripheral immune system by recruiting leukocytes into the CNS [7]. Reactive astrocytosis is a prominent feature in inflammatory condition that occurs during MS and EAE. Experimental evidence suggests that astrocyte activation contributes to a more severe course of MS and EAE characterized by an increased expression of proinflammatory cytokines and chemokines as well as pronounced demyelination [810]. In response to IL-9 treatment, astrocytes increase chemokine production to facilitate $\mathrm{T}$ cell recruitment to the CNS [5]. However, the mechanisms by which astrocyte activation contribute to MS/EAE after IL-9 stimulation are not fully understood.

Long non-coding RNAs (lncRNAs) have been defined as transcripts of $>200$ nucleotides without protein coding capacity. Although genomic studies indicated that approximately twothirds of genomic DNA are pervasively transcribed, less than $2 \%$ of mRNAs are ultimately translated into proteins $[11,12]$. Based on the position of lncRNA in the genome relative to protein-coding genes, lncRNAs are divided into five categories: sense, antisense, intronic, intergenic and bidirectional [13]. LncRNAs are widely involved in various biological and physiological processes including chromatin remodeling, gene transcription, RNA splicing and protein transport, and directly linked to human diseases including various cancers, and neurological disorders [14-16]. It has been reported that lncRNAs regulate the gene expression in the immune system and in autoimmune disease $[17,18]$. However, the roles of lncRNAs in the process of MS/EAE are still unclear.

In the present study, we analyzed the lncRNA and mRNA expression landscape of the brain tissues from EAE mice and activated astrocytes stimulated by IL-9 in vitro. Our results suggest the potential roles of lncRNAs in regulating astrocyte functions during the process of EAE.

\section{Materials and Methods}

Animal ethics statement

All experimental procedures described in the present study were conducted according to the Provision and General Recommendation of the Chinese Laboratory Association. The protocol was approved by the Institutional Animal Care and Use Committee of Xuzhou Medical University. The study was approved by the Ethical Committee for Xuzhou Medical University.

Animal model of EAE

C57BL/6 mice were obtained from Nanjing University Laboratory Animal Center. Mice were housed in clean plastic cages with the temperature of $25 \pm 1 \stackrel{\circ}{\circ} \mathrm{C}$ and humidity of 55-65\% and maintained under specific 


\section{Cellular Physiology Cell Physiol Biochem 2018;45:1986-1998 \begin{tabular}{l|l|l} 
and Biochemistry Published 10.1159/000487975 & $\begin{array}{l}\text { C } 2018 \text { The Author(s). Published by S. Karger AG, Basel } \\
\text { www.karger.com/cpb }\end{array}$
\end{tabular}}

Liu et al.: Lncrna Profile Analysis in Activated Astrocytes and EAE Mice

pathogen-free conditions. EAE models were induced according to our previously published procedure [19]. Briefly, 6- to 8-wk-old C57BL/6 female mice randomly divided into two groups ( $\mathrm{n}=10$ each group): One was injected by s.c. immunization with $200 \mu \mathrm{g} \mathrm{MOG}_{35-55}$ peptide emulsified in complete Freund's adjuvant (CFA, Sigma) containing $5 \mathrm{mg} / \mathrm{ml}$ heat-killed mycobacterium tuberculosis (H37Ra strain, Difco). In addition, 200 ng of pertussis toxin (Invitrogen) in PBS was i.p. injected on day 0 and day 2 after MOG $_{35-55}$ treatment. Mice were monitored daily for clinical signs of EAE and graded on a scale of increasing severity from 0 to 5 : 0 , no clinical signs; 1 , limp tail; 2, paraparesis (weakness, incomplete paralysis of 1 or 2 hind limbs); 3, paraplegia (complete paralysis of 2 hind limbs); 4, paraplegia with fore limb weakness or paralysis; and 5, moribund state or death. Another group was injected with the same volume of PBS, as negative control (NC). Mice were sacrificed after anesthetization by i.p. injection of a mixture of ketamine $(120 \mathrm{mg} / \mathrm{kg})$ and xylazine $(60 \mathrm{mg} / \mathrm{kg})$.

\section{Primary mouse astrocyte cultures}

Primary astrocytes from 0- to 1-day-old C57BL/6 mice were established as previously described [19]. Briefly, the cerebral cortices freed of meninges were dissected, minced and digested. After being washed twice in Dulbecco's Modified Eagle Medium F12 (DMEM/F12) containing 10\% fetal bovine serum (FBS) and antibiotics, the cells were filtrated through a $75 \mu \mathrm{m}$ cell strainer and transferred to culture flasks pre-coated with $1 \mathrm{mg} / \mathrm{ml}$ poly-L-lysine (Sigma) and cultured at $37^{\circ} \mathrm{C}$ with $5 \% \mathrm{CO}_{2}$. At complete confluence, flasks were shaken on an orbital shaker for $1 \mathrm{~h}\left(150 \mathrm{rpm}\right.$ at $\left.37^{\circ} \mathrm{C}\right)$. The cultures were passed for 3 or 4 passages, and glial fibrillary acidic protein (GFAP, astrocytic marker) expression was evaluated via immunofluorescence assay.

\section{Histopathology}

Mice were perfusion-fixed with $4 \%$ paraformaldehyde in $0.1 \mathrm{M}$ sodium phosphate buffer ( $\mathrm{pH} 7.4$ ) under anesthesia. Brains and spinal cords were removed quickly and further fixed with the same fixation solution overnight at $4{ }^{\circ} \mathrm{C}$. The histological evaluation of brains and spinal cord tissues was performed on $4 \mu \mathrm{m}$ paraffin-embedded sections stained with hematoxylin and eosin (H\&E) or luxol fast blue (LFB) to assess inflammation or demyelination under microscopy. Ultrathin sections of spinal cord tissues were stained with uranyl acetate and lead citrate, and the ultrastructural changes were observed under electron microscopy (EM).

\section{RNA extraction}

Total RNA was isolated from the brain tissues of mice and the primary astrocytes according to manufactures instructions. RNA quantity and quality was measured using NanoDrop ND-1000 spectrophotometer (Thermo Fisher Scientific). The RNA integrity of each sample was assessed using standard denaturing agarose gel electrophoresis.

Determination of IncRNA and mRNA profiles in EAE mice and primary astrocytes

The expression profiles of IncRNAs and mRNAs in brain tissues of EAE mice and activated astrocytes were detected using Mouse LncRNA Microarray v2.0 (8 x 60K, Arraystar) by Kangchen Bio-tech (Shanghai, China), which includes 31, 423 lncRNA probes and 25, 376 coding transcripts probes. Microarray assays were also performed using pooled plasma, blood, liver, heart or cell samples [20, 21]. So RNAs from the pooled brain tissues from the control or EAE mice (4 mice in each group) and RNAs from astrocytes (DMEM/ F12 control and IL-9 treatment) underwent microarray analysis. The acquired raw array images were processed by Agilent Feature Extraction software (version 11.0.1.1) and then normalized and analyzed by the GeneSpring GX v12.0 software package (Agilent Technologies). Differentially expressed lncRNAs and mRNAs were then identified through fold-change as well as P values calculated with $t$-test. The threshold for up- and down-regulation was fold change $>2.0$ and p value $<0.05$. Afterwards, Hierarchical Clustering was performed to display the distinguishable lncRNAs and mRNAs expression patterns among the samples.

\section{Real-time PCR assay}

The total RNA from the brain tissues of mice and cultured astrocytes was extracted with TRIzol reagent (Invitrogen). First-strand cDNAs were generated using PrimeScriptTM RT reagent kit (TaKaRa, Japan), and SYBR Premix Ex TaqTM based on real-time PCR (TaKaRa) were used to analyze the relative expression levels of the selected IncRNAs. The relative gene expression was calculated using the $2^{-\Delta \Delta} \mathrm{CT}$ method. The primers are listed in Table 1. 
Functional group analysis

Gene ontology (GO) and KEGG analysis were applied to determine the roles of differentially expressed mRNAs in biological pathways using the standard enrichment computation method. The $p$ value (Hypergeometric-P value) denotes the significance of the pathway correlated to the conditions. The recommend $\mathrm{p}$-value cut-off is 0.05 .

\section{Statistical analysis \\ Data were presented as mean \pm SD. Statistical significance of differences between groups was analyzed by nonparametric tests ( $\mathrm{K}$ independent samples test) or one-way analysis of variance (ANOVA) when more than two groups were compared. GraphPad Prism 5.0 for Microsoft Windows was used to plot all graphs. The criterion for statistical significance was $\mathrm{P}<0.05$.}

\section{Results}

Inflammatory cell infiltration and severe demyelination in EAE mice

The EAE model has been widely used in the investigation of the mechanisms underlying MS. Here, we also found that $\mathrm{MOG}_{35-55}$ peptide induced EAE mice showed increasing severity of clinical signs from scale 1 to 4 as time goes on (Fig. 1A). We then analyzed brain and spinal cord tissues from EAE mice on day 20 after $\mathrm{MOG}_{35 \text { - }}$ ${ }_{55}$ immunization (exhibiting clinical scale 4) by staining with hematoxylin and eosin (HE) and luxol fast blue (LFB), respectively. The results of HE staining showed that the EAE mice brain sections and spinal cord sections developed

Table 1. Primers for verification of lncRNAs by qRT-PCR

\begin{tabular}{|c|c|c|}
\hline Primer Name & Sequence (5' to $\left.3^{\prime}\right)$ & Length (bp) \\
\hline ENSMUST00000119467 & Fw TGCTAACGCAGCCTCTACTCG & 198 \\
\hline AK033297 & $\begin{array}{l}\text { Fw GGCACAGAAGCATTTAGAGCG } \\
\text { Rev GGTTAGCAGTTACTTCCTTTCCTCA }\end{array}$ & 231 \\
\hline ENSMUST00000119913 & $\begin{array}{l}\text { Fw ATCATCAAACCCGAAAGGAGC } \\
\text { Rev AGTCACTGAAGCAGGGCAAGA }\end{array}$ & 145 \\
\hline ENSMUST00000075251 & $\begin{array}{l}\text { Fw TGAAGATTAAGTTTCGTGAAGAACC } \\
\text { Rev TTGTTTCTATGACCATTGGAATCC }\end{array}$ & 244 \\
\hline ENSMUST00000062533 & $\begin{array}{l}\text { Fw TTTGACGGTTGTGATAGGAAGTTTG } \\
\text { Rev AGAGCTTGGGTGGGTGTATGATT }\end{array}$ & 129 \\
\hline ENSMUST00000137239 & $\begin{array}{l}\text { Fw TCTGCTCATAGGAACTCTTGCTATC } \\
\text { Rev CCTTGGAGAAATGGCTGACTT }\end{array}$ & 130 \\
\hline ENSMUST00000156666 & $\begin{array}{l}\text { Fw CCGTGGAAGAACTACTGAACAAGC } \\
\text { Rev GCCTCATTCATCCCAAACTCG }\end{array}$ & 182 \\
\hline ENSMUST00000145143 & $\begin{array}{l}\text { Fw AGGGACCAAGACCAGAAGGAA } \\
\text { Rev GGAATGTGAGGTATCTAAGGGAAGTA }\end{array}$ & 147 \\
\hline ENSMUST00000134472 & $\begin{array}{c}\text { Fw CACTGGCAGCAGGTCCATCTA } \\
\text { Rev TCTTGCTAGTCTTGTCATTCCTGG }\end{array}$ & 248 \\
\hline uc008aid.1 & $\begin{array}{l}\text { Fw CCTGGCACTTTAACTTAGATGAGATT } \\
\text { Rev GGTAGTAGTTGATGGATGGCTTGA }\end{array}$ & 258 \\
\hline uc008rso.1 & $\begin{array}{l}\text { Fw ACATCCAACAGCCCTCAGACA } \\
\text { Rev TCGTCAAGAGCGACAGCAAAC }\end{array}$ & 298 \\
\hline ENSMUST00000149952 & $\begin{array}{l}\text { Fw GCCTCTGCCCAGTTACTTGGAG } \\
\text { Rev CAGTTTGCCATCTGGTTACCCTAC }\end{array}$ & 157 \\
\hline
\end{tabular}

Fig. 1. Identification of EAE mouse model and cultured primary mouse astrocytes. (A) The clinical symptom of EAE mice was scored daily as described in materials and methods. ( $\mathrm{n}=10$ mice per group). (B) Paraffin sections of the brain tissues in EAE on day 20 after $\mathrm{MOG}_{35-55}$ immunization (average clinical scale 4) were stained with H\&E to determine inflammatory cell infiltrations (black arrows, $\mathrm{n}=4$ mice). (C) Sections of the spinal cords in EAE mice stained with H\&E. Scale bars, $50 \mu \mathrm{m}$. (D)

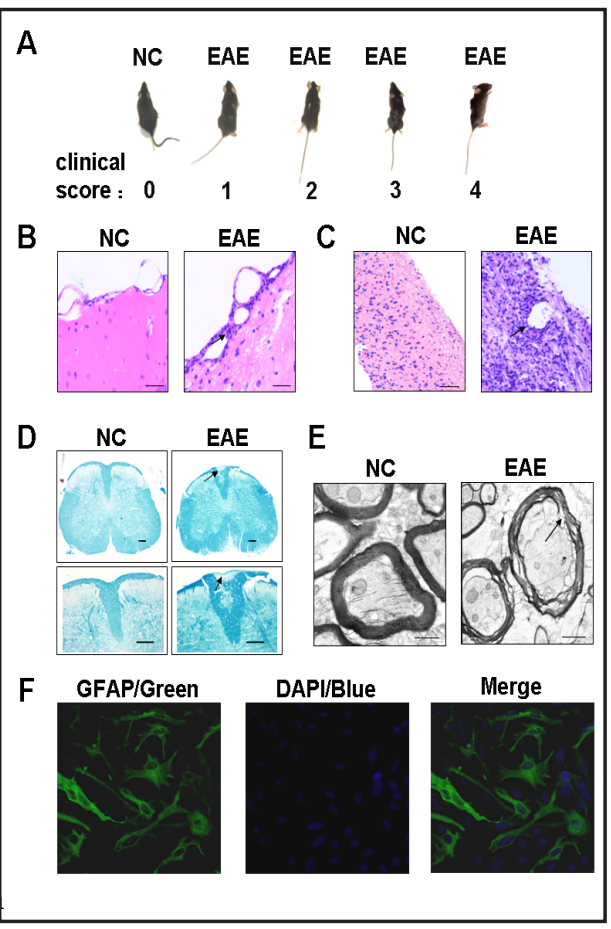
Spinal cord sections of EAE mice exhibiting as demyelination with luxol fast blue (LFB) staining (black arrows), whereas no demyelination was seen in the negative control (NC) group. Scale bars, $50 \mu \mathrm{m}$. (E) Spinal cords of the mice with EAE (average clinical scale 4) under electron microscopy (EM) exhibited loose and disrupted myelination (arrow). Scale bars, $1 \mu \mathrm{m}$. (F) Immunofluorescence for GFAP in cultured primary mouse astrocytes. The cell nuclei were stained by DAPI.

\section{KARGER}


Fig. 2. Scatter plot comparing global lncRNA or mRNA gene expression profiles in vivo and in vitro. IncRNA expression profiles (A) and mRNA expression profiles (B) changed in the brain tissues of EAE mice (clinical scale 1 and scale 4) and NC mice. IncRNA (C) and mRNA (D) expression profiles in the activated astrocytes induced by IL- 9 (at $3 \mathrm{~h}$ and $6 \mathrm{~h}$ ) and DMEM/ F12 control group. The values of $\mathrm{X}$ axis and Y-axis in the scatter-plot were the normalized signal values of each sample (log 2 scaled). The green lines are fold change lines (the default fold change value given is 2.0). The IncRNAs and mRNA above the top green line and below the bottom green line indicate $>$ 2.0 -fold change, compared to the control.

prominent inflammatory cell infiltration, which did not occur in PBS-treated mice (Fig. 1B and C). Furthermore, LFB staining demonstrated that the spinal cords of EAE mice had severe demyelination (Fig. 1D). Specifically, the myelin sheaths of the EAE mice were ruptured and disintegrated under EM (Fig. 1E). For astrocyte isolation, at least GFAP ${ }^{+}$ cells occupied 95\% in cultured mouse primary astrocytes under Fluorescence microscope (Fig. 1F).

\section{Differential LncRNA and mRNA expression in the brain tissues from EAE mice and primary} mouse astrocytes treated with $I L-9$

To detect differentially expressed IncRNAs in the brain tissues of EAE mice and activated mouse primary astrocytes, we performed a genome-wide analysis of IncRNA and mRNA expression in the brain tissues of EAE mice (clinical scale 1 and scale 4) and matched NC mice. Meantime, we also analyzed the IncRNA and mRNA expression profiles of non-activated astrocytes (DMEM/F12 control group) and activated astrocytes induced by IL-9 ( $50 \mathrm{ng} / \mathrm{ml}$, at $3 \mathrm{~h}$ and $6 \mathrm{~h}$ ). We first obtained a graphical overview of the expression signatures of lncRNAs and mRNAs by using scatter plot analyses, which showed that a large number of lncRNAs and mRNAs were differentially expressed in EAE and NC mice (Fig. 2A and B). Similarly, many differentially expressed lncRNAs and mRNAs were observed between the activated astrocytes and DMEM control (Fig. 2C and D).

We then further analyzed differential expression of IncRNAs and mRNAs with fold change $>2$ (the criteria q-value $<0.05$ ) in EAE mice or activated astrocytes compared with NC mice or DMEM control, respectively. Results displayed that 1, 850 lncRNAs and 1, 642 mRNAs were up-regulated, and 1, $450 \mathrm{lncRNAs}$ and 1,608 mRNAs were down-regulated in the brain tissues of EAE mice (Fig. 3A). Meanwhile, 1, $584 \mathrm{lncRNAs}$ and 1, $435 \mathrm{mRNAs}$ were up-regulated, and 2, 164 lncRNAs and 1, 897 mRNAs were down-regulated in the astrocytes stimulated by IL-9 stimulation for $3 \mathrm{~h}$ and $6 \mathrm{~h}$ (Fig. $3 \mathrm{~B}$ ). Notably, there were 2 lncRNAs co-up-regulated and 3 IncRNAs co-down-regulated both in the brain tissues from EAE mice and in the activated 
Fig. 3. Differentially expressed lncRNAs and mRNAs in EAE mice or the activated astrocytes. (A) and (B) Analysis of numbers of significantly expressed IncRNAs and mRNAs. (C) and (D) Overlapping and differentially expressed lncRNAs both in vivo and in vitro. (E) and (F) Overlapping and differentially expressed mRNAs both in vivo and in vitro.

astrocytes (Table 2; Fig. 3C and D). Interestingly, the 5 differentially co-expressed lncRNAs in vivo and in vitro are belong to intergenic lncRNAs, and regulate the ER calcium flux kinetics, zinc finger protein, cell apoptosis and multifunctional serine/threonine protein phosphatases. Similarly, there were 7 mRNAs coup-regulated and 2 mRNAs co-down-regulated both in the brain tissues from EAE mice and in activated astrocytes (Table3; Fig. 3E and F). The list of the top 20 differentially expressed lncRNAs in vivo and in vitro identified by microarray analysis is shown in Table 4-7. Furthermore, the top 20 up-regulated and downregulated mRNA transcripts in vivo and in vitro are displayed in Table 8-11, respectively.

Importantly, some inflammatory cytokines, including CXCL10 (IP-10), CCL2, CXCL2 and TNF were detected in the up-regulated mRNAs in vivo (clinical scale 1 and scale 4, Table 8 ) or in vitro (at $3 \mathrm{~h}$ and $6 \mathrm{~h}$ induced by IL-9, Table 10). These data suggest that MS/EAE is associated with the changes of IncRNAs and mRNAs in the brain tissues and the activated astrocytes.

\section{Validation of the microarray data using real-time PCR}

To further validate the accuracy of microarray data, we randomly selected 12 IncRNAs from the differentially expressed lncRNAs, including 6 that were co-up-regulated (ENSMUST00000119467, AK033297, ENSMUST00000119913, ENSMUST00000075251, ENSMUST00000062533 and ENSMUST00000137239) and 6 co-down-regulated (ENSMUST00000156666, ENSMUST00000145143, ENSMUST00000134472, uc008rso.1, uc008aid.1 and ENSMUST00000149952), and detected their expression through real-time PCR assay. As shown in Fig. 4A, ENSMUST00000119467, AK033297 and ENSMUST00000119913 expressionweresignificantlyincreased,andENSMUST00000156666, 
Fig. 4. Real-time PCR validation of differentially co-expressed lncRNAs in EAE mice or the activated astrocytes. 12 IncRNAs were randomly chosen for real-time PCR validation in vivo and in vitro. (A) The expressions of IncRNA ENSMUST00000119467, AK033297 and ENSMUST00000119913 were significantly increased in the brain tissues of EAE mice with clinical scale 1 and in scale 4, meanwhile, ENSMUST00000156666, ENSMUST00000145143 and ENSMUST00000134472 expression were obviously decreased in EAE mice. ${ }^{* *} \mathrm{P}<0.01$, ${ }^{* * *} \mathrm{P}<0.001$ vs $\mathrm{NC}$ ( $\mathrm{n}=3$ mice per group). (B) The expressions of IncRNA ENSMUST00000075251, ENSMUST00000062533andENSMUST00000137239 were significantly up-regulated in activated astrocytes stimulated by IL- 9 for $3 \mathrm{~h}$ and $6 \mathrm{~h}$, while uc008rso.1, uc008aid.1, ENSMUST00000149952 were markedly down-regulated in activated astrocytes. ${ }^{*} \mathrm{P}<0.05$, ${ }^{* *} \mathrm{P}<0.01,{ }^{* * *} \mathrm{P}<0.001$ vs DMEM. Error bars represent means \pm SD.

ENSMUST00000145143

and ENSMUST00000134472 expression were significantly decreased in the brain tissues of EAE mice with clinical scale 1 and scale 4, compared with NC control. The expression levels of the ENSMUST00000075251, ENSMUST00000062533 and ENSMUST00000137239 were markedly up-regulated, and uc008rso.1, uc008aid.1, ENSMUST00000149952 were significantly down-regulated in activated astrocytes stimulating with IL-9 for $3 \mathrm{~h}$ and $6 \mathrm{~h}$, compared with DMEM control (Fig. 4B). Overall, these results confirmed the accuracy of microarray data obtained from analysis of pooled samples.

\section{Class distribution of changed IncRNAs}

According to the relative location of lncRNA to coding genes, we analyzed class distribution of differentially co-expressed LncRNAs. Among the co-expressed lncRNAs in the brain tissues of EAE mice with clinical scale 1 and scale 4 , intergenic lncRNAs were the largest category along with 270 upregulated and 150 down-regulated IncRNAs. Secondly, among antisense IncRNAs 80 were up-regulated and 57 were down-regulated. Thirdly, among sense IncRNAs 67 were upregulated and 20 were down-regulated. Bidirectional IncRNAs were the smallest

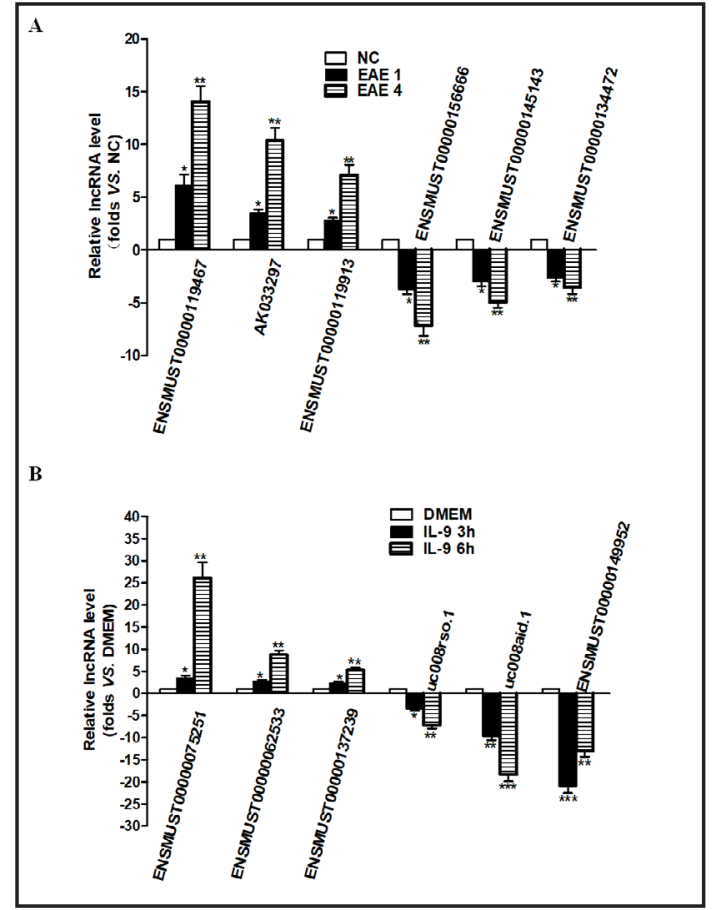

Table 4. Top 20 overlapped and up-regulated IncRNAs of the brain tissues from EAE mice with clinical scale 1 and 4

\begin{tabular}{lccc}
\hline IncRNA seqname & RNA length & \multicolumn{2}{c}{ up-regulated fold } \\
& Scale1/NC & Scale 4/NC \\
\hline ENSMUST00000119669 & 663 & 7.85589 & 10.23717 \\
uc008use.1 & 3335 & 8.610238 & 9.648406 \\
ENSMUST00000119467 & 1274 & 4.3121204 & 9.547864 \\
AK033297 & 3620 & 2.6846502 & 8.161776 \\
NR_033450 & 2051 & 8.633687 & 8.022282 \\
uc0095pu.1 & 1729 & 22.872778 & 7.0451984 \\
AK045052 & 1766 & 6.6805325 & 7.0031447 \\
uc008rso.1 & 778 & 6.1572304 & 6.500624 \\
MM9LINCRNAEXON11637+ & 1180 & 5.5882964 & 6.351306 \\
uc007rdz.1 & 1529 & 6.1322365 & 6.0852413 \\
AK008216 & 1208 & 10.930726 & 6.014869 \\
ENSMUST00000135792 & 424 & 6.7050333 & 5.8834567 \\
AK141778 & 1953 & 3.3443995 & 5.8223014 \\
ENSMUST00000037976 & 913 & 5.596207 & 5.7913294 \\
AK136771 & 2813 & 4.8559976 & 5.628442 \\
humanlincRNA2345- & 11309 & 10.116245 & 5.574991 \\
ENSMUST00000160565 & 1414 & 5.6017933 & 5.5404854 \\
ENSMUST00000153719 & 726 & 5.106356 & 5.521596 \\
uc.150+ & 262 & 5.8136806 & 5.2537236 \\
AK133322 & 3838 & 4.8335 & 5.206456 \\
\hline
\end{tabular}

Table 5. Top 20 overlapped and down-regulated IncRNAs of the brain tissues from EAE mice with clinical scale 1 and 4

\begin{tabular}{lccc}
\hline IncRNA seqname & RNA length & \multicolumn{2}{c}{ down-regulated fold } \\
Scale1/NC & Scale 4/NC \\
\hline ENSMUST00000163836 & 420 & -40.030895 & -37.572098 \\
uc009iwa. & 832 & -7.5178285 & -7.8960743 \\
AK020153 & 710 & -6.851927 & -5.964112 \\
CN673952 & 517 & -5.260042 & -5.617605 \\
ENSMUST00000156666 & 1319 & -3.937882 & -5.388637 \\
AK082604 & 2334 & -2.9760792 & -4.963089 \\
AK006093 & 270 & -5.834375 & -4.864372 \\
AF346502 & 325 & -4.7329516 & -4.8320174 \\
AK019721 & 538 & -4.6150265 & -4.790841 \\
AK042092 & 2251 & -4.2353625 & -4.676630 \\
AK015350 & 1005 & -4.1139274 & -4.5655036 \\
BC051648 & 1091 & -5.0416775 & -4.529922 \\
ENSMUST00000145143 & 560 & -2.9116006 & -4.4599357 \\
uc.394+ & 202 & -3.5275517 & -4.4599357 \\
AK034340 & 3369 & -12.039054 & -4.446819 \\
ENSMUS00000120738 & 412 & -4.793811 & -4.224718 \\
AK131760 & 1316 & -3.0181768 & -4.16362 \\
AK033086 & 2497 & -3.5453696 & -3.9964097 \\
ENSMUST00000148691 & 710 & -4.1512413 & -3.933257 \\
AK155134 & 3476 & -3.5343256 & -3.8350103 \\
\hline
\end{tabular}




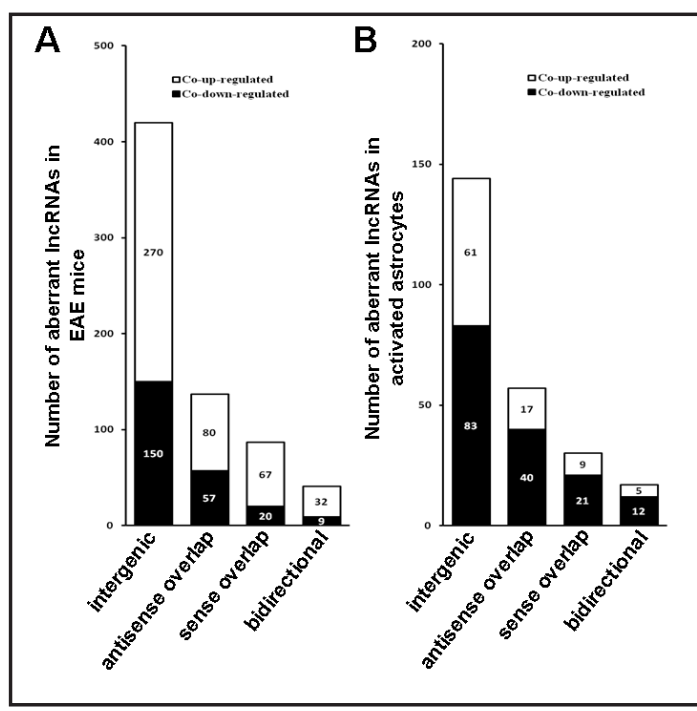

Fig. 5. Distribution of various types of IncRNAs. Co-expressed numbers of intergenic lncRNAs, antisense overlap lncRNAs, sense overlap lncRNAs and bidirectional IncRNAs were analyzed in the brain tissues of EAE mice with clinical scale 1 and scale 4 (A) and in activated astrocytes stimulated by IL-9 for $3 \mathrm{~h}$ and $6 \mathrm{~h}(\mathrm{~B})$.

category including 32 up-regulated and 9 down-regulated lncRNAs (Fig. 5A). Similarly, as shown in Fig. 5B, among the coexpressed lncRNAs in the activated mouse primary astrocytes stimulated with IL-9 for $3 \mathrm{~h}$ and $6 \mathrm{~h}$, intergenic lncRNAs were still the largest category, including 61upregulated and 83 down-regulated lncRNAs. The other differentially co-expressed IncRNAs included 57 antisense lncRNAs (17 up-regulated and 40 down-regulated), 30 sense lncRNAs (9 up-regulated and 21 down-regulated), and 17 bidirectional IncRNAs (5 up-regulated and 12 downregulated).

\section{Go and KEGG pathway analysis}

To further explore potential molecular mechanism in MS, we ran GO and KEGG Pathway analysis of differentially expressed genes in the brain tissues of EAE mice and activated astrocytes. GO analysis indicated that differentially co-up-regulated transcripts were biological regulation, metabolism regulation and biological process regulation in the brain tissues of EAE mice and activated astrocytes (Fig. 6A and B). Furthermore, differentially co-down-regulated genes were mainly associated with cell development in the brain tissues of EAE mice and activated astrocytes (Fig. 6C and D).
Table 6. Top 20 overlapped and up-regulated lncRNAs in mouse astrocytes stimulated with IL-9 $(50 \mathrm{ng} / \mathrm{ml})$ for $3 \mathrm{~h}$ and $6 \mathrm{~h}$ in vitro

\begin{tabular}{|c|c|c|c|}
\hline \multirow[b]{2}{*}{ IncRNA seqname } & \multirow[b]{2}{*}{ RNA length } & \multicolumn{2}{|c|}{ up-regulated fold } \\
\hline & & $3 \mathrm{~h} / \mathrm{DMEM}$ & $6 \mathrm{~h} / \mathrm{DMEM}$ \\
\hline ENSMUST00000075251 & 522 & 3.9349897 & 40.166023 \\
\hline ENSMUST00000062533 & 606 & 2.0524364 & 12.538993 \\
\hline AK083446 & 2226 & 2.2753801 & 12.145436 \\
\hline humanlincRNA0889+ & 23120 & 3.0090966 & 8.693425 \\
\hline MM9LINCRNAEXON11983+ & 1278 & 2.2759817 & 7.63561962 \\
\hline ENSMUST00000165402 & 699 & 3.0055168 & 4.8639135 \\
\hline ENSMUST00000137239 & 1475 & 2.0873954 & 4.690183 \\
\hline AK083633 & 1745 & 2.104949 & 4.188388 \\
\hline uc. $480+$ & 202 & 2.6271284 & 4.010122 \\
\hline uc008ydi. 1 & 605 & 3.2122352 & 3.9657302 \\
\hline uc008ajj.1 & 364 & 3.383075 & 3.900578 \\
\hline MM9LINCRNAEXON12120+ & 360 & 2.537424 & 3.8664494 \\
\hline AK020497 & 977 & 2.0829475 & 3.858925 \\
\hline AK014172 & 761 & 2.4132385 & 3.7605712 \\
\hline AK146536 & 2999 & 2.801698 & 3.7465243 \\
\hline MM9LINCRNAEXON11883- & 1673 & 7.5292897 & 3.7110455 \\
\hline ENSMUST00000129149 & 549 & 4.7973676 & 3.6296542 \\
\hline uc. $30+$ & 243 & 2.941384 & 3.5964377 \\
\hline AK014724 & 1450 & 2.270314 & 3.5466068 \\
\hline uc008ich.1 & 1068 & 2.039356 & 3.5054522 \\
\hline
\end{tabular}

Table 7. Top 20 overlapped and down-regulated IncRNAs in mouse astrocytes stimulated with IL-9 (50 $\mathrm{ng} / \mathrm{ml}$ ) for $3 \mathrm{~h}$ and $6 \mathrm{~h}$ in vitro

\begin{tabular}{|c|c|c|c|}
\hline \multirow[b]{2}{*}{ IncRNA seqname } & \multirow[b]{2}{*}{ RNA length } & \multicolumn{2}{|c|}{ down-regulated fold } \\
\hline & & $3 \mathrm{~h} / \mathrm{DMEM}$ & $6 \mathrm{~h} /$ DMEM \\
\hline MM9LINCRNAEXON10644- & 529 & -117.604935 & -99.0716 \\
\hline AK136795 & 2457 & -20.417713 & -20.625822 \\
\hline uc008aid.1 & 2654 & -12.142941 & -20.602228 \\
\hline AK047651 & 1505 & -16.56007 & -16.410059 \\
\hline ENSMUST00000149952 & 435 & -34.908175 & -15.565921 \\
\hline AK039597 & 918 & -12.962295 & -14.543182 \\
\hline AK044317 & 1433 & -11.480068 & -12.880182 \\
\hline MM9LINCRNAEXON10750+ & 270 & -12.000128 & -12.189643 \\
\hline ENSMUST00000167046 & 1189 & -11.106656 & -11.7804575 \\
\hline AK140223 & 2651 & -2.0036714 & -11.718122 \\
\hline uc007ztt.1 & 4087 & -7.7851024 & -8.734577 \\
\hline uc008rso.1 & 778 & -3.6056058 & -8.127705 \\
\hline uc008Ill1.1 & 1020 & -6.8208704 & -7.652747 \\
\hline uc008jnj.1 & 2334 & -5.3359036 & -7.1349945 \\
\hline AK156979 & 3729 & -2.875295 & -7.0227046 \\
\hline MM9LINCRNAEXON10159+ & 424 & -2.4457703 & -6.8156323 \\
\hline AK146287 & 865 & -4.4910707 & -6.699524 \\
\hline ENSMUST 00000098624 & 1317 & -6.066518 & -6.6513 \\
\hline MM9LINCRNAEXON10379+ & 406 & -3.5663848 & -6.1227465 \\
\hline AK020959 & 532 & -2.0639613 & -6.068381 \\
\hline
\end{tabular}

Table 8. Top 20 overlapped and up-regulated mRNAs in the brain tissues of EAE mice with clinical scale 1 and 4

\begin{tabular}{lccc}
\hline \multirow{2}{*}{ Gene symbol } & Gene length & \multicolumn{2}{c}{ up-regulated fold } \\
& & Scale 1/NC & Scale 4/NC \\
\hline Ttr & 1237 & 335.11658 & 68.372696 \\
Cxcl10 & 1063 & 20.973085 & 33.02361 \\
S100a8 & 392 & 48.44693 & 29.814625 \\
Cfb & 2767 & 36.640877 & 22.740374 \\
Tmem102 & 1941 & 4.4589195 & 17.689392 \\
Ch25h & 1365 & 12.644377 & 10.278557 \\
Olfr91 & 939 & 8.076411 & 9.904047 \\
1110059M19Rik & 1123 & 23.963148 & 9.76958 \\
Ifi204 & 2302 & 8.120821 & 8.638965 \\
Ccl5 & 579 & 6.5959787 & 8.330466 \\
Gm14548 & 2130 & 14.441691 & 8.216907 \\
Ccl12 & 537 & 6.719245 & 8.077436 \\
Lcn2 & 853 & 12.209477 & 7.716298 \\
H2-Q8 & 1183 & 6.778891 & 7.393144 \\
Frmd6 & 4695 & 3.224566 & 7.015356 \\
Ifi205 & 1613 & 9.347171 & 6.800729 \\
Ccl2 & 806 & 3.637598 & 6.4596 \\
Apobec3 & 2459 & 3.7751663 & 6.212857 \\
Zbp1 & 781 & 8.288381 & 6.201317 \\
Cd74 & 1239 & 10.382884 & 6.1446013 \\
\hline
\end{tabular}


Table 9. Top 20 overlapped and down-regulated mRNAs in the brain tissues of EAE mice with clinical scale 1 and 4

\begin{tabular}{lccc}
\hline $\begin{array}{c}\text { Gene } \\
\text { symbol }\end{array}$ & Gene length & \multicolumn{2}{c}{ down-regulated fold } \\
& & Scale 1/NC & Scale 4/NC \\
\hline Cux2 & 5133 & -2.0224001 & -50.37911 \\
Cd300lb & 2880 & -15.030037 & -15.786247 \\
Hoxa9 & 2272 & -8.497262 & -11.213646 \\
Dact3 & 2828 & -14.50526 & -7.4577813 \\
Cep97 & 7904 & -6.039256 & -6.9138865 \\
Ccdc136 & 3507 & -4.752726 & -6.1138263 \\
Calm3 & 2233 & -13.898376 & -4.7527223 \\
Ehmt1 & 5070 & -2.5776749 & -4.6222963 \\
Gstt3 & 1873 & -4.3256702 & -4.578973 \\
Dock1 & 6815 & -4.7299695 & -4.3923683 \\
Stambpl1 & 1978 & -14.772 & -4.329778 \\
Tgfbr2 & 4728 & -5.0039163 & -4.315667 \\
Il17rc & 2272 & -3.4610384 & -4.3087068 \\
Men1 & 2644 & -5.994871 & -4.152673 \\
Neur14 & 5203 & -5.317888 & -4.1331754 \\
Lepr & 3407 & -3.9306805 & -4.1284456 \\
Gm11710 & 852 & -2.6119237 & -4.0417027 \\
Stk25 & 3254 & -6.3022923 & -4.024722 \\
Rsad2 & 3785 & -3.831459 & -4.024232 \\
Cacnb2 & 2970 & -4.6388793 & -3.9718573 \\
\hline
\end{tabular}

Table 10. Top 20 overlapped and up-regulated mRNAs in mouse astrocytes stimulated with IL-9 (50 $\mathrm{ng} / \mathrm{ml}$ ) for $3 \mathrm{~h}$ and $6 \mathrm{~h}$ in vitro

\begin{tabular}{lccc}
\hline Gene symbol & Gene length & \multicolumn{2}{c}{ up-regulated fold } \\
& & $3 \mathrm{~h} / \mathrm{DMEM}$ & $6 \mathrm{~h} / \mathrm{DMEM}$ \\
\hline Ccl2 & 806 & 3.1756024 & 9.821831 \\
Nrxn1 & 9040 & 2.2285407 & 9.118354 \\
Ereg & 4136 & 2.6865191 & 6.7909007 \\
Ccl7 & 912 & 2.5141113 & 6.3414073 \\
Fpr1 & 1332 & 6.2122507 & 5.5888267 \\
Lrrc55 & 2900 & 2.7651134 & 5.2560782 \\
Bcl11b & 7923 & 2.5492954 & 5.0328083 \\
Cxcl2 & 1083 & 4.2408137 & 4.948825 \\
Klrb1c & 2641 & 3.2704635 & 4.811274 \\
Tnf & 1619 & 5.9117227 & 4.6778245 \\
Chm & 4867 & 2.8186088 & 4.4003916 \\
Rnf20 & 4159 & 3.6893559 & 4.212892 \\
6430704M03Rik & 3854 & 2.0068235 & 4.1690726 \\
Gbp6 & 5548 & 2.0744424 & 3.9634035 \\
Ak4 & 4894 & 2.5767694 & 3.9619064 \\
Tcstv3 & 878 & 6.379969 & 3.6716871 \\
4930415L06Rik & 2618 & 2.5779061 & 3.394951 \\
Ajap1 & 3067 & 4.3802185 & 3.2806315 \\
Vsig8 & 1814 & 2.9814172 & 3.2300596 \\
Itch & 5214 & 3.3478744 & 3.229839 \\
\hline
\end{tabular}

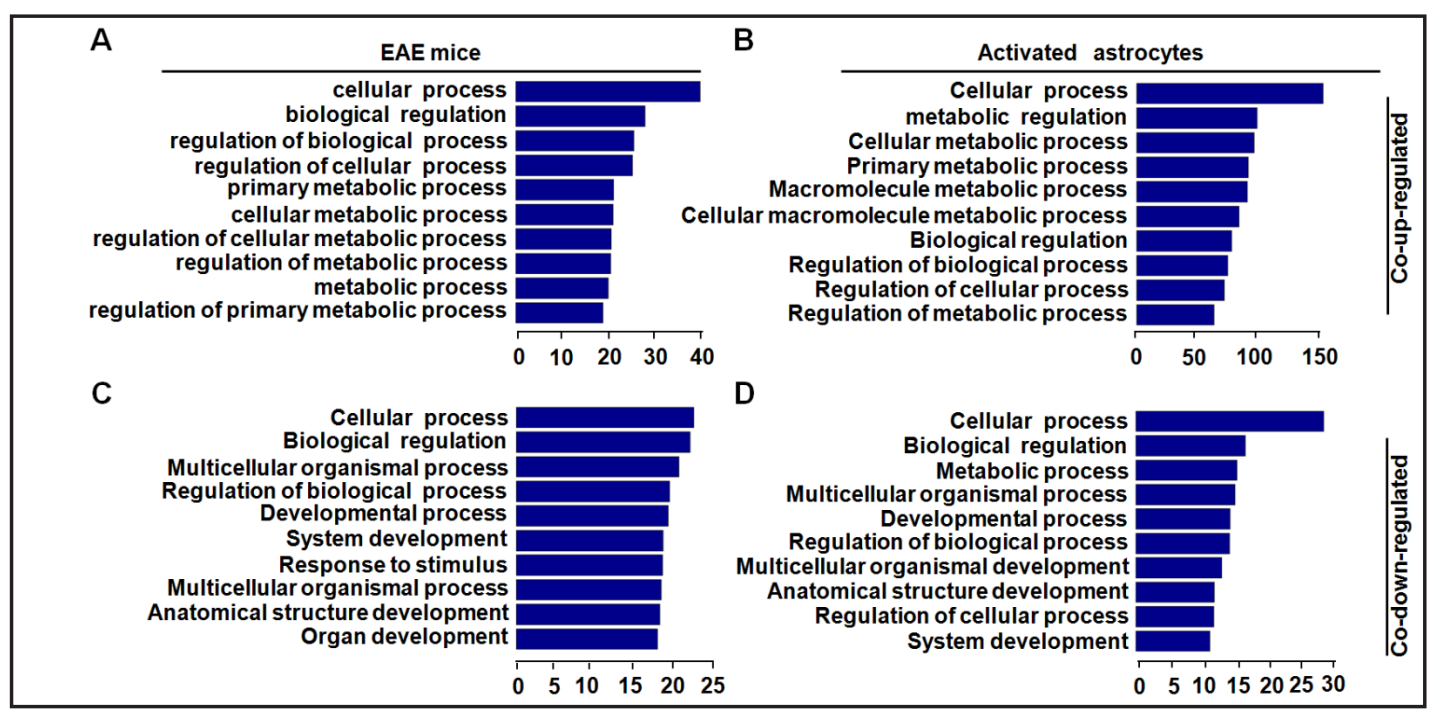

Fig. 6. Biological functions of differential overlap genes with fold changes $>2.0$. (A and B) The significant biological process, cellular component and molecular function of co-up-regulated genes or co-downregulated genes were shown in the brain tissues of EAE mice with clinical scale 1 and scale 4. (C and D) Significantly enriched molecular function, biological process and cellular component of co-up-regulated genes or co-down-regulated genes were identified in the activated astrocytes stimulated by IL- 9 for $3 \mathrm{~h}$ and $6 \mathrm{~h}$.

KEGG Pathway analysis showed that 40 pathways were significantly enriched among differentially expressed genes. Co-up-regulated genes in the brain tissues of EAE mice with clinical scale 1 and scale 4 were related to glutamatergic synapases, calcium signaling pathways, retrograde endocannabinoid signaling, adherens junctions, leukocyte transendothelial migration and so on (Fig. 7A). The co-down-regulated genes in EAE mice were involved in the renin-angiotensin system, African trypanosomiasis, malaria, neuroactive ligand-receptor interaction, etc (Fig. 7B). Similarly, KEGG Pathway results indicated that the co-up-regulated genes in the activated astrocytes stimulated by IL-9 for 3 $\mathrm{h}$ and $6 \mathrm{~h}$ were involved in PI3K-Akt signaling pathway, focal adhesion, adherens junctions, regulation of actin cytoskeleton, etc (Fig. 7C). Furthermore, the co-down-regulated genes in 


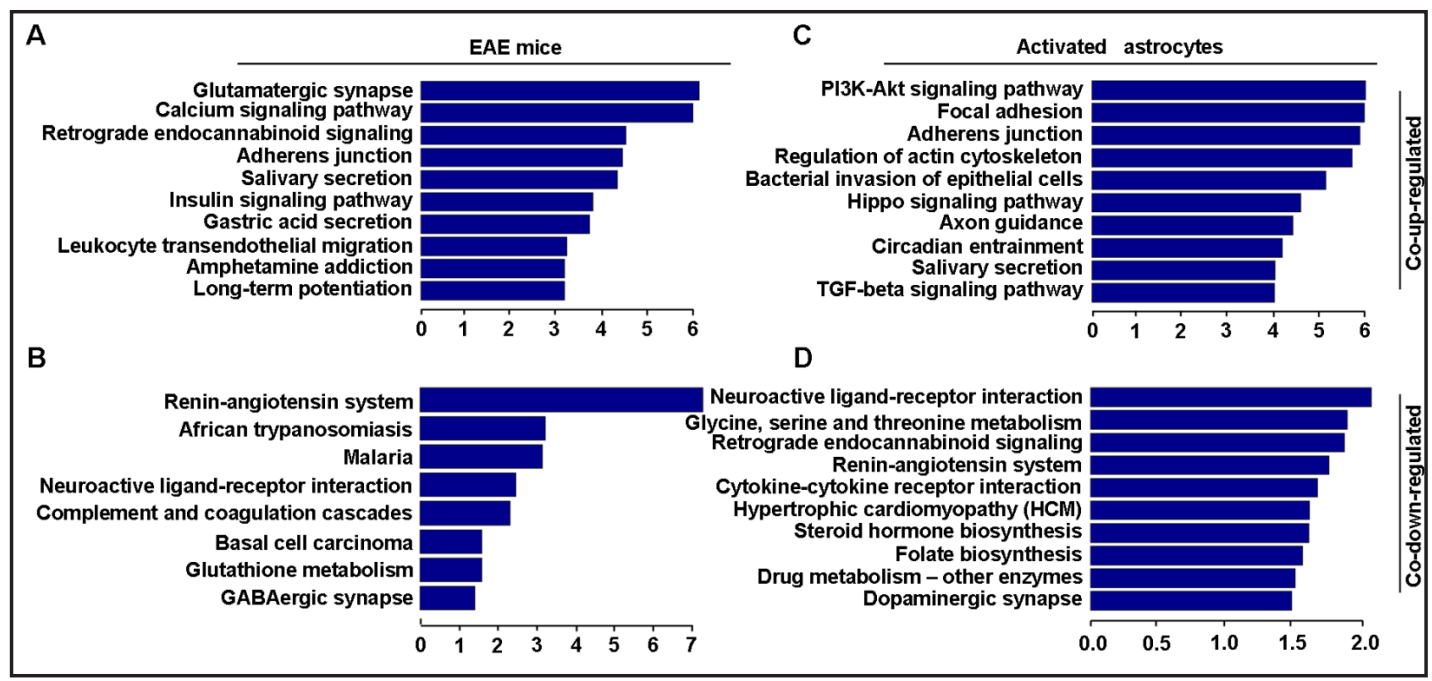

Fig. 7. KEGG Pathway analysis of differential overlap genes with fold changes $>2.0$. (A) The significant pathways for co-up-regulated genes in the brain tissues of EAE mice with clinical scale 1 and scale 4. (B) The remarked pathways for co-down-regulated genes in the brain tissues of EAE mice. (C) The significant pathways for co-up-regulated genes in astrocytes stimulated by IL-9 for $3 \mathrm{~h}$ and $6 \mathrm{~h}$. (D) The significant pathways for co-down-regulated genes in astrocytes stimulated by IL- 9 for $3 \mathrm{~h}$ and $6 \mathrm{~h}$.

the activated astrocytes were involved in neuroactive ligand-receptor interaction, glycine, serine and threonine metabolism, retrograde endocannabinoid signaling, renin-angiotensin system, etc (Fig. 7D).

\section{Discussion}

MS/EAE is a chronic autoimmune disease of the central nervous system with inflammation, demyelination and axon degradation. Many studies have demonstrated that the blockade of proinflammatory cytokines production in astrocytes can attenuate the damage of MS/EAE [19-21].

Over the past decades, the molecular mechanisms underlying MS have been extensively studied. However, our understanding of the pathophysiological process of MS is still incomplete. In recent years, IncRNAs have received much attention in human diseases. Aberrant expression or function of lncRNAs have been linked to cancer, neurological disorders, and hemolysis, elevated liver enzymes and low platelets (HELLP) syndrome [18, 22, 23]. Moreover, IncRNAs are important regulators of immune cells differentiation and function, eg. $\mathrm{T}$ cells, dentritic cells, B cells, macrophages and NK cells [24]. Emerging evidence indicates that lncRNAs not only participate in autoimmune diseases including systemic lupus erythematosus (SLE), rheumatoid arthritis (RA) and psoriasis [25-27], but also play important roles in CNS development, homeostasis, stress responses, and plasticity [28]. Recent work points to the role of IncRNAs in oligodendrocyte precursor cell (OPC) differentiation from neural stem cells (NSCs), myelination and remyelination in the CNS [29, 30]. IncRNA (Malat1) displays 
anti-apoptotic and anti-inflammatory roles in microvasculature structure of the brain to reduce ischemic brain injury [31]. However, there is a large gap between the number of existing IncRNAs and their known association with a particular molecular or cellular function in MS/EAE. To date, the functional characterization of lncRNAs during the EAE progression and astrocyte activation has not been carried out systematically. In the present study, we explored the expression profiles of IncRNAs and mRNAs both in the brain tissues of EAE mice and in IL-9-induced astrocytes, analyzed the co-expression of IncRNAs in vivo and in vitro, and inferred their characteristics and possible relations with protein-coding genes.

There were 1, 850 up-regulated lncRNAs and 1, 642 up-regulated mRNAs in the brain tissues of EAE mice. Meanwhile 1, 450 lncRNAs and 1, 608 mRNAs were down-regulated. Furthermore, 1, 584 lncRNAs and 1, 435 mRNAs were up-regulated, and 2, 164 lncRNAs and 1, 897 mRNAs were down-regulated in the astrocytes stimulated by IL- 9 for $3 \mathrm{~h}$ and $6 \mathrm{~h}$. Notably, there were 2 IncRNAs that differentially co-up-regulated and 3 IncRNAs that differentially co-down-regulated both in the brain tissue of EAE mice and in activated astrocytes. There were 7 mRNAs co-up-regulated and 2 mRNAs co-down-regulated both in the brain tissues from EAE mice and in activated astrocytes. Moreover, real-time PCR were performed to verify part of results in EAE mice and in activated astrocytes, which was consistent with the results of lncRNAs microarray. These altered lncRNAs revealed a close association with the levels of inflammatory cytokines secreted by activated astrocytes and severity of EAE mice, suggesting these lncRNAs might provide novel insight into the molecular basis of MS/EAE.

A number of inflammation-related genes were dramatically co-up-regulated in EAE mice and in activated astrocytes, such as cxcl10 (IP-10), cxcl2, ccl2 and tnf. Furthermore, some mRNAs including complement factor B, Transthyretin, LIM homeobox protein 6, G-protein coupled receptor 84 (Gpr84) and Fatty acid binding protein 4 were also screened out and remarkably increased both in vivo and in vitro (Table 8 and 10), whose functions are unclear in the brain tissue of EAE mice. Further studies are needed to identify whether they are involved in EAE processing.

It has been established that IncRNAs are involved in the regulation of the immune system, including NF- $\kappa \mathrm{B}$ signaling, anti-viral response, $\mathrm{CD} 4^{+}$and $\mathrm{CD} 8^{+} \mathrm{T}$-cell differentiation and inflammatory response [18]. Based on the GO term enrichment and pathway maps of mRNAs, we found that markedly enriched molecular functions and biological processes of up-regulated gene in EAE mice were mainly involved in metabolism regulation, biological regulation, biological process regulation and inflammation. These findings are consistent with previous studies showing that the infiltration of immune cells and inflammations play an important role in the induction and maintenance of MS/EAE [19].

\section{Conclusion}

In summary, our results revealed that IncRNA transcripts were highly enriched and thousands of lncRNAs were differentially expressed in the brain tissues of EAE mice and in activated astrocytes. These IncRNAs were observed to share intergenic, sense overlap, antisense overlap, or bidirectional mRNAs in genome, which may regulate their related protein-genes expression and play key roles in the pathogenesis of EAE. Further studies on these lncRNAs are required to clarify their molecular and cellular functions and determine whether they can serve as potential therapeutic targets in EAE.

\section{Acknowledgements}

This work was supported by the Natural Science Foundation of Jiangsu Province (BK20151168 to Liu, BK20141136 to Zhou), Dean Special Foundation of Xuzhou Medical University (2012KJZ10 to Liu), Xuzhou Technology Bureau Foundation (KC14SH074 to 


\section{Cellular Physiology Cell Physiol Biochem 2018;45:1986-1998 \begin{tabular}{ll|l} 
and Biochemistry Published online: March 08, 2018 & $\begin{array}{l}\text { (C) } 2018 \text { The Author(s). Published by S. Karger AG, Basel } \\
\text { www.karger.com/cpb }\end{array}$
\end{tabular}}

Liu et al.: Lncrna Profile Analysis in Activated Astrocytes and EAE Mice

Liu), Jiangsu Key Laboratory of Brain Disease Bioinformation to Liu (2015KF01), Jiangsu Key Laboratory Foundation of New Drug Research and Clinical Pharmacy to Liu, the Priority Academic Program Development of Jiangsu Higher Education Institutions (2014 PAPD), the National Natural Science Foundation of China (81501762 to Pan, 81461138036 to Sun), the Natural Science Foundation of the Jiangsu Higher Education Institutions (15KJB310025 to Pan) and the Graduate Innovation Program in Science and Technology of Jiangsu Province (KYLX16_1132 to Wang). Moreover, we would like to thank Jessica M. Meves (University of California San Diego) for reviewing this manuscript.

\section{Disclosure Statement}

None of the authors have any conflicts of interest in this study.

\section{References}

1 Steinman L: Immunology of relapse and remission in multiple sclerosis. Annu Rev Immunol 2014;32:257281.

2 Hauser SL, Oksenberg JR: The neurobiology of multiple sclerosis: Genes, inflammation, and neurodegeneration. Neuron 2006;52:61-76.

3 Jager A, Dardalhon V, Sobel RA, Bettelli E, Kuchroo VK: Th1, th17, and th9 effector cells induce experimental autoimmune encephalomyelitis with different pathological phenotypes. J Immunol 2009;183:7169-7177.

4 Li H, Nourbakhsh B, Ciric B, Zhang GX, Rostami A: Neutralization of il-9 ameliorates experimental autoimmune encephalomyelitis by decreasing the effector $t$ cell population. J Immunol 2010;185:40954100.

-5 Zhou Y, Sonobe Y, Akahori T, Jin S, Kawanokuchi J, Noda M, Iwakura Y, Mizuno T, Suzumura A: Il-9 promotes th17 cell migration into the central nervous system via cc chemokine ligand-20 produced by astrocytes. J Immunol 2011;186:4415-4421.

6 Sofroniew MV, Vinters HV: Astrocytes: Biology and pathology. Acta Neuropathol 2009;119:7-35.

7 Wilson EH, Weninger W, Hunter CA: Trafficking of immune cells in the central nervous system. J Clin Invest 2010;120:1368-1379.

8 Haroon F, Drogemuller K, Handel U, Brunn A, Reinhold D, Nishanth G, Mueller W, Trautwein C, Ernst M, Deckert M, Schluter D: Gp130-dependent astrocytic survival is critical for the control of autoimmune central nervous system inflammation. J Immunol 2011;186:6521-6531.

-9 Skripuletz T, Hackstette D, Bauer K, Gudi V, Pul R, Voss E, Berger K, Kipp M, Baumgartner W, Stangel M: Astrocytes regulate myelin clearance through recruitment of microglia during cuprizone-induced demyelination. Brain 2012;136:147-167.

10 Giraud SN, Caron CM, Pham-Dinh D, Kitabgi P, Nicot AB: Estradiol inhibits ongoing autoimmune neuroinflammation and $\mathrm{nf}$ b-dependent ccl2 expression in reactive astrocytes. Proc Natl Acad Sci U S A 2010;107:8416-8421.

11 Lee JT: Epigenetic regulation by long noncoding rnas. Science 2012;338:1435-1439.

$>12$ Fatica A, Bozzoni I: Long non-coding rnas: New players in cell differentiation and development. Nat Rev Genet 2014;15:7-21.

13 Mercer TR, Dinger ME, Mattick JS: Long non-coding rnas: Insights into functions. Nat Rev Genet 2009;10:155-159.

14 Gibb EA, Vucic EA, Enfield KS, Stewart GL, Lonergan KM, Kennett JY, Becker-Santos DD, MacAulay CE, Lam S, Brown CJ, Lam WL: Human cancer long non-coding rna transcriptomes. PloS one 2011;6:e25915.

15 Qureshi IA, Mehler MF: Emerging roles of non-coding rnas in brain evolution, development, plasticity and disease. Nat Rev Neurosci 2012;13:528-541.

16 Johnson R: Long non-coding rnas in huntington's disease neurodegeneration. Neurobiol Dis 2012;46:245254.

17 Atianand MK, Fitzgerald KA: Long non-coding rnas and control of gene expression in the immune system. Trends in Mol Med 2014;20:623-631. 


\section{Cellular Physiology Cell Physiol Biochem 2018;45:1986-1998

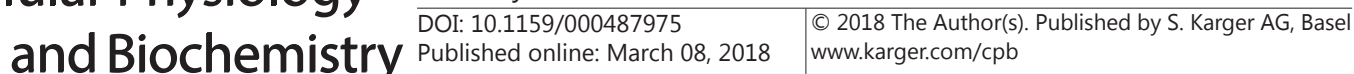

18 Hrdlickova B, Kumar V, Kanduri K, Zhernakova DV, Tripathi S, Karjalainen J, Lund RJ, Li Y, Ullah U, Modderman R, Abdulahad W, Lahdesmaki H, Franke L, Lahesmaa R, Wijmenga C, Withoff S: Expression profiles of long non-coding rnas located in autoimmune disease-associated regions reveal immune celltype specificity. Genome Med 2014;6:88.

19 Liu XM, He FX, Pang RR, Zhao D, Qiu W, Shan K, Zhang J, Lu YL, Li Y, Wang YW: Interleukin-17 (il17)-induced microrna 873 (mir-873) contributes to the pathogenesis of experimental autoimmune encephalomyelitis by targeting a20 ubiquitin-editing enzyme. J Biol Chem 2014;289:28971-28986.

-20 Kang Z, Altuntas CZ, Gulen MF, Liu C, Giltiay N, Qin H, Liu L, Qian W, Ransohoff RM, Bergmann C, Stohlman S, Tuohy VK, Li X: Astrocyte-restricted ablation of interleukin-17-induced act1-mediated signaling ameliorates autoimmune encephalomyelitis. Immunity 2010;32:414-425.

-21 Yun HM, Park KR, Kim EC, Hong JT: Prdx6 controls multiple sclerosis by suppressing inflammation and blood brain barrier disruption. Oncotarget 2015;6:20875-20884.

-22 Prensner JR, Iyer MK, Sahu A, Asangani IA, Cao Q Patel L, Vergara IA, Davicioni E, Erho N, Ghadessi M, Jenkins RB, Triche TJ, Malik R, Bedenis R, McGregor N, Ma T, Chen W, Han S, Jing X, Cao X, Wang X, Chandler B, Yan W, Siddiqui J, Kunju LP, Dhanasekaran SM, Pienta KJ, Feng FY, Chinnaiyan AM: The long noncoding rna schlap1 promotes aggressive prostate cancer and antagonizes the swi/snf complex. Nat Genet 2013;45:1392-1398.

23 Wapinski O, Chang HY: Long noncoding rnas and human disease. Trends Cell Biol 2011;21:354-361.

-24 Fitzgerald KA, Caffrey DR: Long noncoding rnas in innate and adaptive immunity. Curr Opin Immunol 2014;26:140-146.

25 Zhang FF, Wu LL, Qian J, Qu B, Xia SW, La T, Wu YF, Ma JY, Zeng J, Guo Q, Cui Y, Yang WL, Huang JQ, Zhu W, Yao YH, Shen N, Tang YJ: Identification of the long noncoding rna neat1 as a novel inflammatory regulator acting through mapk pathway in human lupus. J Autoimmun 2016;75:96-104.

-26 Wu GC, Pan HF, Leng RX, Wang DG, Li XP, Li XM, Ye DQ: Emerging role of long noncoding rnas in autoimmune diseases. Autoimmun Rev 2015;14:798-805.

27 Sigdel KR, Cheng A, Wang Y, Duan L, Zhang Y: The emerging functions of long noncoding rna in immune cells: Autoimmune diseases. J Immunol Res 2015;2015:848790.

28 Briggs JA, Wolvetang EJ, Mattick JS, Rinn JL, Barry G: Mechanisms of long non-coding rnas in mammalian nervous system development, plasticity, disease, and evolution. Neuron 2015;88:861-877.

29 Dong X, Chen K, Cuevas-Diaz Duran R, You Y, Sloan SA, Zhang Y, Zong S, Cao Q Barres BA, Wu JQ: Comprehensive identification of long non-coding rnas in purified cell types from the brain reveals functional lncrna in opc fate determination. PLoS Genet 2015;11:e1005669.

30 He D, Wang J, Lu Y, Deng Y, Zhao C, Xu L, Chen Y, Hu YC, Zhou W, Lu QR: Lncrna functional networks in oligodendrocytes reveal stage-specific myelination control by an lncol1/suz12 complex in the cns. Neuron 2017;93:362-378.

-31 Zhang X, Tang X, Liu K, Hamblin MH, Yin KJ: Long noncoding rna malat1 regulates cerebrovascular pathologies in ischemic stroke. J Neurosci 2017;37:1797-1806. 